ARTICLE

Received 13 Jan 2014 | Accepted 10 Apr 2014 | Published 27 May 2014

DOI: $10.1038 /$ ncomms4851

OPEN

\title{
Fabrication of crystals from single metal atoms
}

\author{
Nicolas P. E. Barry ${ }^{1}$, Anaïs Pitto-Barry ${ }^{1}$, Ana M. Sanchez ${ }^{2}$, Andrew P. Dove ${ }^{1}$, Richard J. Procter ${ }^{1}$, \\ Joan J. Soldevila-Barreda ${ }^{1}$, Nigel Kirby ${ }^{3}$, Ian Hands-Portman ${ }^{4}$, Corinne J. Smith ${ }^{4}$, Rachel K. O'Reilly ${ }^{1}$, \\ Richard Beanland ${ }^{2} \&$ Peter J. Sadler ${ }^{1}$
}

Metal nanocrystals offer new concepts for the design of nanodevices with a range of potential applications. Currently the formation of metal nanocrystals cannot be controlled at the level of individual atoms. Here we describe a new general method for the fabrication of multi-heteroatom-doped graphitic matrices decorated with very small, ångström-sized, three-dimensional (3D)-metal crystals of defined size. We irradiate boron-rich preciousmetal-encapsulated self-spreading polymer micelles with electrons and produce, in real time, a doped graphitic support on which individual osmium atoms hop and migrate to form 3D-nanocrystals, as small as $15 \AA$ in diameter, within $1 \mathrm{~h}$. Crystal growth can be observed, quantified and controlled in real time. We also synthesize the first examples of mixed ruthenium-osmium 3D-nanocrystals. This technology not only allows the production of ångström-sized homo- and hetero-crystals, but also provides new experimental insight into the dynamics of nanocrystals and pathways for their assembly from single atoms.

\footnotetext{
${ }^{1}$ Department of Chemistry, University of Warwick, Gibbet Hill Road, Coventry CV4 7AL, UK. ${ }^{2}$ Department of Physics, University of Warwick, Gibbet Hill Road, Coventry CV4 7AL, UK. ${ }^{3}$ Australian Synchrotron, 800 Blackburn Road, Clayton, Victoria 3168, Australia. ${ }^{4}$ School of Life Sciences, University of Warwick, Gibbet Hill Road, Coventry CV4 7AL, UK. Correspondence and requests for materials should be addressed to N.P.E.B.

(email: N.Barry@warwick.ac.uk) or to R.B. (email: R.Beanland@warwick.ac.uk) or to P.J.S. (email: P.J.Sadler@warwick.ac.uk).
} 
T ailoring nanoscopic objects is of importance for the production of the materials of the future, for example in medicine, industrial manufacturing, construction and space exploration ${ }^{1-4}$. The potential for use of metal nanocrystals has long been recognized. For example, colloidal gold was first made by Andreas Cassius in 1685 (ref. 5), and notably also by Michael Faraday in 1857 (ref. 6). Since then, a range of techniques has allowed the fabrication of nanocrystals, and these have allowed detailed investigation of their size-dependent properties ${ }^{7}$. The recent emergence of powerful methods such as high-resolution transmission electron microscopy, has enabled the design and the observation of a variety of nanometersized objects, from graphene matrices, to nanoparticles and nanotubes 8,9 .

The process of crystallization, the formation of microscopic periodic arrangements of atoms, ions and molecules, has long been a topic of intense fascination. It is 100 years since William Henry Bragg and his son William Lawrence Bragg revolutionized the characterization of crystalline solids by showing that the atomic structure of a crystal is related to its X-ray diffraction pattern ${ }^{10}$. Understanding the dynamics of crystal formation is also of great interest as the mode of assembly can influence the type of periodic lattice which is formed, and hence the properties of the resulting crystals. However, the direct observation of resolved single atoms, atom-by-atom cluster formation and the crystallization process for nanoscopic crystals is still an experimental frontier ${ }^{1-15}$. Currently, formation of such crystals cannot be controlled at the level of individual atoms ${ }^{16-20}$, and understanding the metal dynamics of nanocrystal formation relies mostly on computation ${ }^{18-20}$.

Here we introduce a new general method for the fabrication of multi-heteroatom-doped graphitic matrices decorated with very small, angstrom-sized, three-dimensional (3D)-metal crystals of defined size. This technology allows experimental observation of crystal assembly from single metal atoms and captures the dynamics of metal cluster formation in real space with atomic precision. We irradiate boron-rich precious metal-encapsulated self-spreading polymer micelles with electrons and produce, in real time, a doped graphitic support on which individual metal atoms hop and migrate to form $3 \mathrm{D}$-nanocrystals, as small as $15 \AA$ in diameter and within $1 \mathrm{~h}$. Crystal growth can be observed, quantified and controlled in real time. Our procedure is exemplified by osmium, the densest of all metals ${ }^{21}$, and gives rise to osmium crystals, which are among the smallest reported ${ }^{22}$, embedded into a B/S-doped graphitic support. As an example of the generality of the procedure, we also synthesize the first examples of mixed ruthenium-osmium 3D-nanocrystals. This technology not only allows the production of angstrom-sized homo- and hetero-crystals, highly novel materials with potentially useful and unusual properties, but also provides new experimental insight into the dynamics of nanocrystals and pathways for their assembly from single atoms.

\section{Results}

Preparation of precursors. Our procedure involved the encapsulation of the organometallic half-sandwich Os ${ }^{\mathrm{II}}$ arene complex [Os( $p$-cymene)(1,2-dicarba-closo-dodecarborane-1,2dithiolate)] (1), a 16-electron complex ${ }^{23}$, which is highly hydrophobic ${ }^{24}$; in the water-soluble amphiphilic triblock copolymer P123 (ref. 25) at ambient temperature for $4 \mathrm{~h}$ to form OsMs micelles (Fig. 1a). The OsMs micelles were fully characterized by a range of techniques, including synchrotron small-angle X-ray scattering (SAXS) experiments (see Methods, Supplementary Figs 1-6, and Supplementary Table 1). These analyses demonstrated that complex 1 and polymer P123 self-assemble in solution to give core/shell micelles with very low-dispersity parameters (0.161), containing $52 \pm 6 \quad$ P123 unimers, along with $52 \pm 11$ complex 1 . They have a core diameter of $9.06 \pm 0.12 \mathrm{~nm}$, and a shell diameter of $6.50 \pm 0.15 \mathrm{~nm}$, giving a total-diameter of $15.56 \pm 0.27 \mathrm{~nm}$. The data are summarized in Supplementary Table 1. OsMs micelles are dispersible in water. They contain a defined number of metal complexes, and are deformable on surfaces since P123 polymer forms stable Langmuir films at ambient temperature $^{26}$. Thus, we deposited aqueous droplets $\left([\mathbf{O s M s}]=1 \mathrm{mg} \mathrm{ml}^{-1}\right)$ onto lacey carbon-coated grids to produce an unsupported film over the grid holes for examination by aberration-corrected high-resolution transmission electron microscopy (HR-TEM).

Formation of a graphitic matrix and Os nanocrystals. We observed structural changes within the Pluronic film upon irradiation with the high-energy electron beam $(80 \mathrm{keV}$; $1.9 \mathrm{pA} \mathrm{cm}^{-2}$ or $7.6 \times 10^{7}$ electrons $\left.\cdot \mathrm{nm}^{-2} \mathrm{~s}^{-1}\right)$. The emergence of atomic ordering within the self-supporting matrix consistent with a turbostratic graphitic structure was apparent within a few minutes, and a highly structured few-layer graphene lattice was evident after $50 \mathrm{~min}$. An analysis of this matrix is shown in Fig. 1. The existence of individual layers of single-sheet graphene-like material at the edges of the material was demonstrated by fast Fourier transform (FFT) analysis (Fig. 1b), while few-layer graphene-like material was also visible (Fig. 1c shows a region with three layers). Along with these structural modifications of the self-supporting polymeric film, a rapid decomposition of the carborane-containing complex $\mathbf{1}$ was also observed (in less than $1 \mathrm{~min}$ ), and Os atoms could be imaged either singly or as smallordered clusters (Fig 1c,e), which is currently at the forefront of such experiments $9,19,27,28$.

Electron energy loss spectroscopy not only confirmed the identity of the Os atoms, but also suggested that boron and sulfur from the carborane ligand in $\mathbf{1}$ are present in the graphitic matrix (Supplementary Fig. 7). Notably, sites with Os clusters (Supplementary Fig. 7c) appear to be close to both high-boron (Supplementary Fig. 7b,e,f) and high-sulfur sites (Supplementary Fig. 7a,d,f). Interestingly, boron and sulfur atoms are also seen throughout the entire matrix suggesting the formation of a multiheteroatom-doped graphitic matrix. After $1 \mathrm{~min}$ of irradiation, clusters of ca. $0.7 \mathrm{~nm}$ were already visible (Fig. 2a). After $5 \mathrm{~min}$ of irradiation, dark areas containing ca. 15 atoms, became more organized and larger (ca. $1.0 \mathrm{~nm}$, Fig. 2b). Further growth of the nanoclusters on the self-supporting matrix was evident after $15 \mathrm{~min}$ of irradiation (Fig. 2c), and after $30 \mathrm{~min}$, osmium metal clusters with diameters of $c a .1 .2-1.6 \mathrm{~nm}$ were visible (Fig. $2 \mathrm{~d}$ ). Growth was observed until ca. $60 \mathrm{~min}$ (Fig. 2), and the nanoclusters were seen to roll on the support in a series of images recorded within a minute (Supplementary Video 1). The movement of energized nanoclusters themselves results in contact with neighbouring particles, and eventual merging. Such cluster merging is illustrated in Supplementary Fig. 8 (also Supplementary Video 2). Interestingly, Zoberbier et al. ${ }^{9}$ reported that clusters of 20-60 Os atoms inside carbon nanotubes continuously change their shape under the influence of an $80 \mathrm{keV}$ electron beam, and bind strongly to the inner surface, weakening $\mathrm{C}-\mathrm{C}$ bonds and promoting gradual removal of carbon atoms.

Measurements of the length of the clusters indicate a linear relationship with time (Fig. $2 \mathrm{~g}$ ). This description is however only indicative owing to the $3 \mathrm{D}$ nature of the nanocrystals (Supplementary Video 3). Significantly, this in situ generation of Os atoms on a self-supporting graphitic matrix produces $3 \mathrm{D}$ 
a
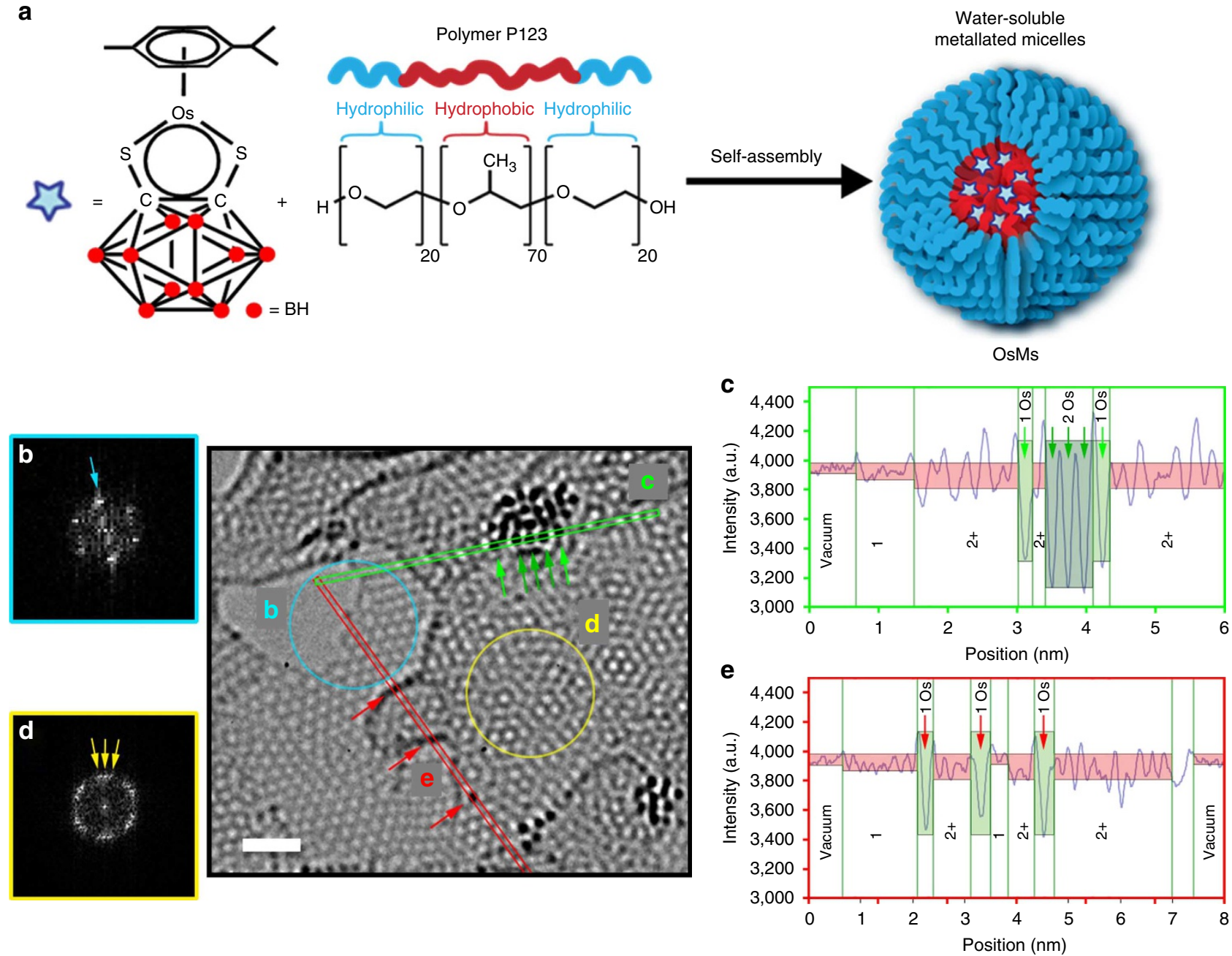

Figure 1 | Micelle formation and analysis after irradiation with electrons. (a) Self-assembly of block copolymer micelles OsMs containing encapsulated osmium carborane complex (b-e): analysis of a typical area after degradation of the micelles by irradiation showing formation of a selfsupporting graphitic matrix and individual osmium atoms (scale bar, $1 \mathrm{~nm}$ ), characterized as follows: (b) Fast Fourier transform corresponding to singlesheet graphene-like material at the edges of the material, blue circle. (c) Intensity plot along the green line; the difference in thickness of the graphitic matrix (single layer versus 2 or more layers) is evident from the range of intensities (pink bands), similarly the difference between single and stacked Os atoms is evident for the cluster of atoms (green arrows), demonstrating its 3D nature. (d) Fast Fourier transform corresponding to a three-layer graphene-like material, yellow circle. (e) Detection of single Os atoms along the red line.

nanoclusters that are crystalline $\mathrm{e}^{29}$. This is illustrated by the fast Fourier transform (FFT) analysis (Fig. $2 \mathrm{~h}$ ) of the ca. $1.5 \mathrm{~nm}$ diameter Os nanocrystal depicted in Fig. 2f. Supplementary Fig. 9 shows a montage of 10 osmium crystals along with their corresponding FFT analyses. In general, the Os crystals do not seem to have a simple hexagonal structure. Supplementary Fig. 10 shows two particles from the montage in Supplementary Fig. 9 with the closest appearance to the hexagonal structure and compares them with simulations. The c-axis view is distinctive, and the a-axis view is a good fit, with the correct interatomic distances. Nevertheless, we believe that most of the images do not match either of these two views, which are not the only ones with views that look relatively simple. The other views have more complex behaviour dependent on particle size and microscope focus. However, the average Os-Os distance measured over 85 different nanocrystals was determined to be $0.257 \pm 0.019 \mathrm{~nm}$, close to that in crystals of bulk osmium metal $(0.27048 \mathrm{~nm}$ as the nearest neighbor distance at $293.15 \mathrm{~K})^{30,31}$. No change in Os-Os distance was observed during nanocrystal growth, as shown by the narrow standard deviation for the average Os-Os distance calculated from these 85 nanocrystals of various sizes (width between 1.5 and $2 \mathrm{~nm}$ ).

Mixed Os/Ru nanocrystals. To demonstrate the generality of this new technology, we encapsulated 1 mol equivalent of osmium complex 1 and $1 \mathrm{~mol}$ equivalent of its ruthenium analogue [Ru(p-cymene)(1,2-dicarba-closo-dodecarborane-1,2-dithiolate)] in RuOsMs micelles, spread them onto a lacey carbon grid and irradiated them with the electron beam in a similar procedure as described above. Again, it was possible to observe metal atom migration and nanocrystal formation on a timescale of $c a .1 \mathrm{~h}$ (Fig. 3).

The composition of the crystals was analyzed by a combination of scanning-TEM and energy-dispersive X-ray analysis at the single particle level (Supplementary Fig. 11); this clearly demonstrated their hetero-metallic Ru/Os nature. Interestingly, the $\mathrm{Ru} / \mathrm{Os}$ molar ratios determined for 55 different crystals $(0.91 \pm 0.07 / 1.13 \pm 0.07)$ are very close to the initial $\mathrm{Ru} / \mathrm{Os}$ ratio in the RuOsMs micelles determined by inductively coupled plasma 

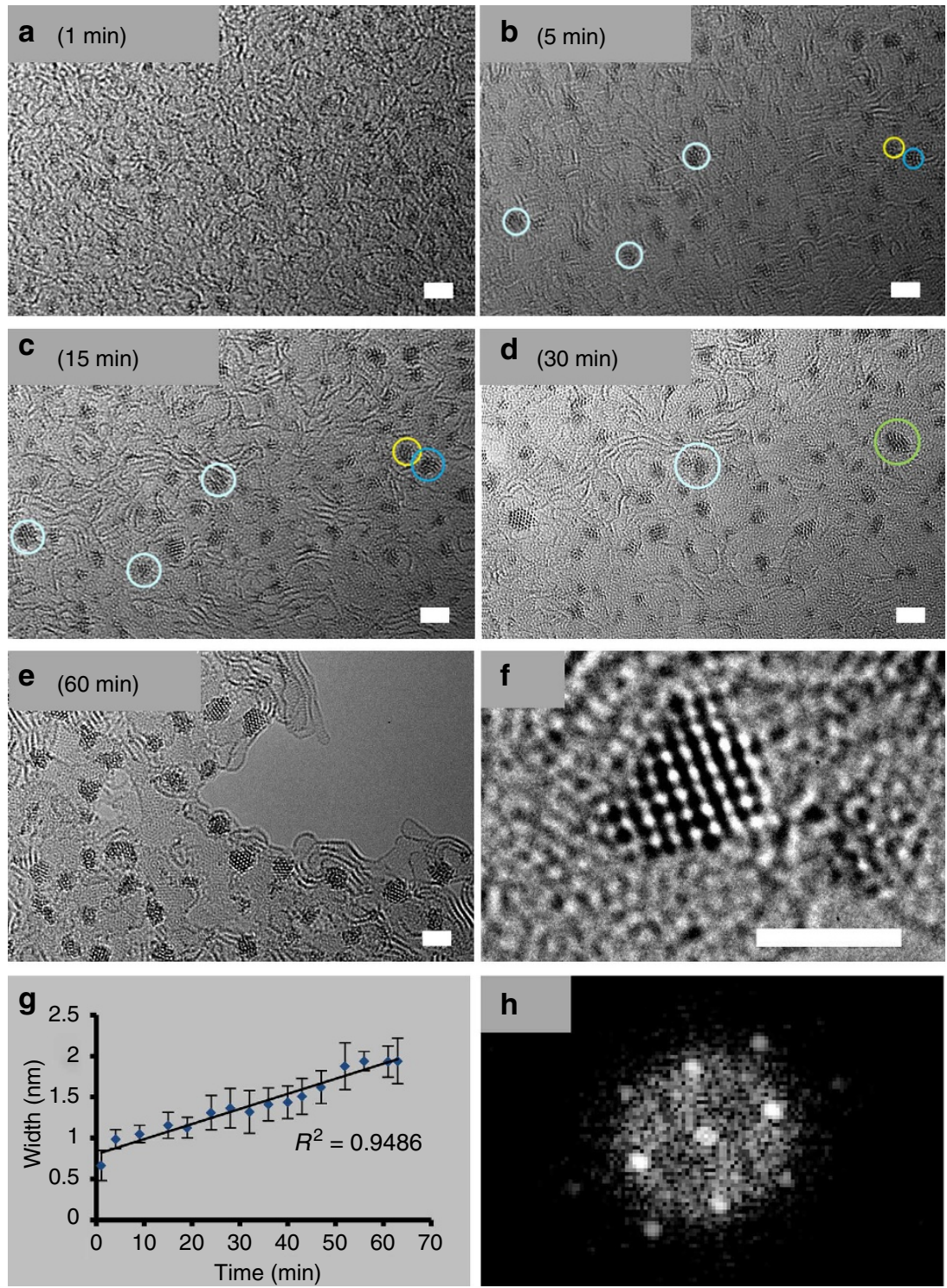

Figure 2 | Time-dependent formation of Os nanocrystals on the graphitic matrix. (a-d) Migration of small clusters and their coalescence (for example, clusters in yellow and dark blue circles merge to give crystal in green circle) over a period 1-30 min; scale bars, $2 \mathrm{~nm}$. (e) The abundance of nanocrystals after 60 min. (f) Typical example of an Os crystal of ca. $1.5 \mathrm{~nm}$, formed after 30 min of irradiation, scale bar, $1.5 \mathrm{~nm}$. (g) Width of the clusters/crystals versus time. The error bars are the standard deviations from the mean for measurements of the width of at least 20 clusters at each timepoint. (h) Fast Fourier transform analysis of the nanocrystal shown in $\mathbf{f}$.

mass spectrometry (ICP-MS) $(0.83 \pm 0.10 / 1.11 \pm 0.13)$. This suggests that the atomic percentage of each metal in the nanocrystals can be readily tuned by varying the ratio of metal complexes encapsulated in the micelles, opening-up new perspectives for the design of hetero-metal nanocrystals with defined size and composition.

\section{Discussion}

We investigated the pattern and the rate of single Os movement by following the positions of a single Os atom over $200 \mathrm{~s}$ (20 frames with 10 s between each frame; Supplementary Fig. 12c, Supplementary Fig. 12d). The positions were extracted from the movie shown in Supplementary Video 4. Attempts were made to correlate the 'hopping' pattern with three different mechanisms: edge-to-edge, bridge-to-bridge and hole-to-hole hopping over the hexagonal graphitic matrix (Supplementary Fig. 12a,b). The closest fit corresponds to Os atoms moving from bridge-to-bridge (that is, sitting between a pair of two adjacent atoms) at a rate of $0.0089 \pm 0.0016 \mathrm{~nm} \mathrm{~s}^{-1}$. Such a bridge localization of Os atoms is apparent in some HR-TEM pictures (Supplementary Fig. 13), and is consistent with the favoured location of Pt atoms adsorbed on boron-doped graphene predicted by calculations ${ }^{18}$. Moreover, extrinsic point and line defects, such as foreign atoms at different positions, strongly modify the charge distribution and the electronic structure of graphene ${ }^{32}$. Density functional theory calculations have also suggested that graphitic boron dopants induce a deficiency of charge in graphene sheets, while sulfur doping of graphene is of particular interest as the resulting materials are expected to have a wider band gap than pure graphene ${ }^{33}$. Boron-doped graphene films show a large number of Stone-Wales defects ${ }^{34}$, while calculations indicate that sulfur doping induces a large local curvature that tends to increase the local reactivity ${ }^{35}$. Metal atoms have a high affinity for non-perfect and strained regions of point defects in graphene created by electron irradiation and annealing ${ }^{36}$. Thus, boron and sulfur 
a

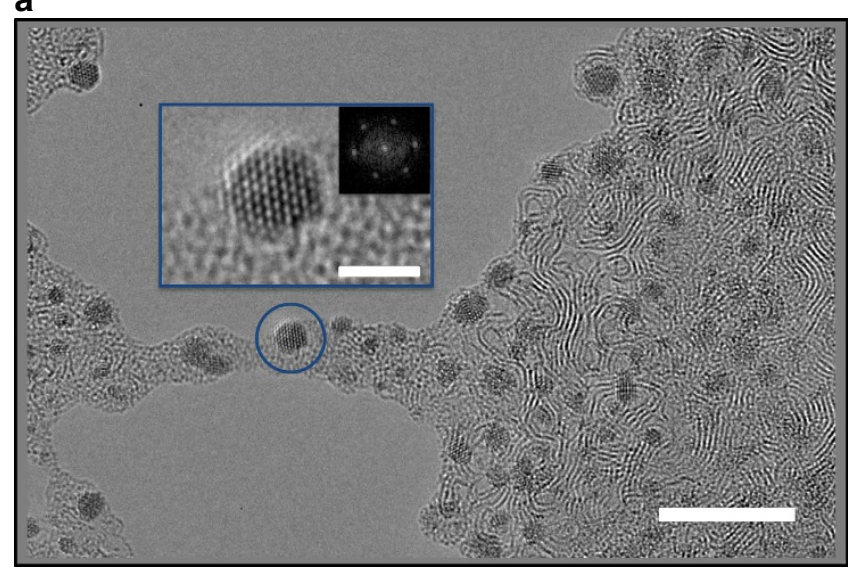

b

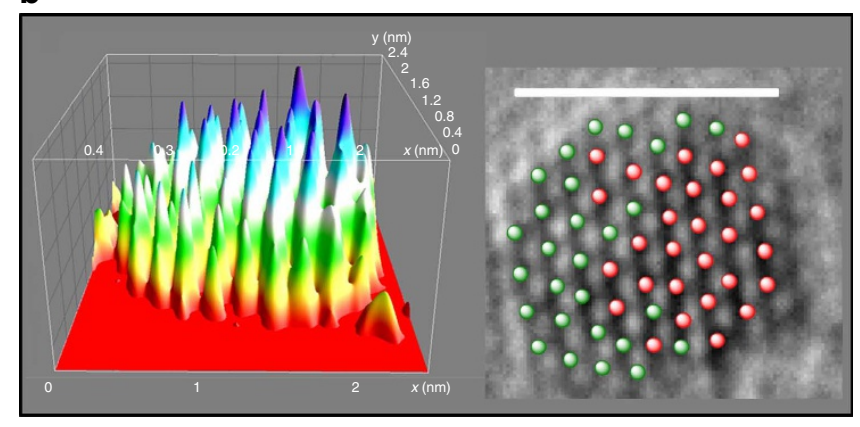

Figure 3 | Ru-Os 3D-nanocrystals from RuOsMs micelles. (a) An array of mixed metal nanocrystals on the graphitic support formed after $60 \mathrm{~min}$ of irradiation (scale bar, $10 \mathrm{~nm}$ ), with enlargement of the blue circled crystal showing atomic resolution (scale bar, $2 \mathrm{~nm}$ ) and fast Fourier transform analysis of the hexagonal mixed metal crystal. The fast Fourier transform analysis of the hexagonal mixed metal crystal is also shown in the enlargement. (b) 3D projection (left) of the same crystal showing the difference of contrast between $\mathrm{Ru}$, Os and the background (each peak corresponds to an atom, and the height/intensity of the peaks is dependent on the atomic TEM contrast; arbitrary colours), also depicted as 29 red (Os) and 28 green (Ru) balls on the 2D projection (right, scale bar, $2.1 \mathrm{~nm}$ ). The presence of Ru and Os atoms was confirmed by a combination of scanning-TEM and energy-dispersive $\mathbf{X}$-ray analysis.

centres and the resultant defects might act as centres, which attract Os atoms. This was confirmed by HR-TEM images showing that Os atoms are located in disturbed zones of the hexagonal pattern of the matrix (Supplementary Fig. 14). Individual Os atoms were also observed at step edges (Fig. 1d); similar observations have been reported for $\mathrm{Au}$ and $\mathrm{Pt}$ atoms on graphene layers 37 .

Such atom-by-atom fabrication of $\mathrm{di}$ - and poly-atomic molecules led to the rapid assembly of Os atoms into clusters. Figure 4 shows the atom-by-atom formation of osmium molecules, clusters made up of a few Os atoms and nanocrystals. This appears to be the first observation of atom-by-atom fabrication of nanocrystals.

In conclusion, our synthetic technology provides a new route to the in situ generation of an unsupported graphitic matrix together with metal atoms, which can migrate to form crystals. The method not only allows the multi-doping of the graphitic matrix, in this case with both boron and sulfur, but also captures the dynamics of metal cluster formation in real space with atomic precision. Indeed, this first report of the use of block copolymer micelles to encapsulate metal complexes for in situ reduction to metal atoms and formation of nanocrystals has allowed the dynamics of formation of metal nanocrystals to be observed, all the way from single atoms to molecules, clusters and then nanocrystals. This method has been exemplified by the production of a graphitic matrix doped with sulfur and boron, decorated with ångström-sized crystals of osmium, opening-up possible new perspectives for the design of nanoscopic highly-dense and pressure-resistant materials. The technology can readily be extended to expand the range of dopants in the supporting graphitic matrix, for example by replacing sulfur with selenium. It is also facile to fabricate a range of homo- and hetero-metal angstrom-size nanocrystals. We have illustrated this for mixed ruthenium-osmium crystals. Other combinations that might be readily accessible include $\mathrm{Pd}, \mathrm{Rh}, \mathrm{Ir}$ and $\mathrm{Au}$, as the synthesis of the precursor carborane complexes of these metals is feasible. There is also wide scope for adapting this polymer-encapsulated metal complex synthetic procedure by variation of the block copolymer. The possibility of creating individual vacancies at desired locations in carbon nanotubes using electron beams has been recently demonstrated ${ }^{38}$, and might also be combined with our procedure to allow the grafting of Os nanocrystals onto specific hotspots. Our synthetic route, which gives rise to a selfsupporting graphitic matrix, also offers attractive possibilities for studying the formation of multi-heteroatom-doped-graphitic sheets and the influence of dopants and defects without influence from the underlying support grids (for example, copper), a problem which often complicates the interpretation of metal deposition experiments. A combination of new experiments and computation will be necessary in future work for fully understanding the detailed mechanisms of doping, hopping, cluster rolling and coalescence. Finally, these nanocrystals may contribute to conceptual advances in the design of a new range of nanodevices, for example, for information storage, electronic circuitry, chemosensing and catalysis.

\section{Methods}

Materials. The preparation of the complex [Os $(p$-cym)(1,2-dicarba-closododecaborane-1,2-dilthiolate)] (1) was based on a previous report ${ }^{23}$. The triblock copolymer P123 [poly(ethylene glycol)-block-poly(propylene glycol)-blockpoly(ethylene glycol)] was purchased from Sigma-Aldrich and used as received. Anhydrous tetrahydrofuran (Aldrich) was used. Pure water (18.2 M $\Omega$ ) was collected from a Purelab UHQ USF Elga system. Holey carbon grids with 200 mesh and lacey carbon grids were purchased from Quantifoil Micro Tools Gmbh and Elektron Technology UK Ltd, respectively and used as received.

Synthesis of OsMs and OsRuMs. A tetrahydrofuran solution ( $1 \mathrm{ml})$ of complex 1 $\left(5 \mathrm{mg} \mathrm{ml}^{-1}\right)$ was added to an aqueous solution $(10 \mathrm{ml})$ of polymer P123

$\left(5 \mathrm{mg} \mathrm{ml}^{-1}\right)$ and the resultant mixture was stirred at ambient temperature for $4 \mathrm{~h}$. The solution was then dialyzed to remove the tetrahydrofuran (molecular weight cutoff $=1000 \mathrm{Da}$ ), for $48 \mathrm{~h}$, and then freeze-dried. A similar procedure was used for synthesizing OsRuMs with $1 \mathrm{~mol}$ equivalent of $1,1 \mathrm{~mol}$ equivalent of the $\mathrm{Ru}$ analogue and $1 \mathrm{~mol}$ equivalent of polymer P123.

Characterization of the micelles OsMs and P123Ms. Dynamic light scattering (DLS) experiments (Supplementary Fig. 2) unambiguously demonstrated that polymer P123 and complex 1 self-assemble in aqueous solution. Encapsulation decreased the size of P123Ms micelles from $19.6 \pm 1.80 \mathrm{~nm}$ (hydrodynamic diameter, $D_{\mathrm{h}}$ ) to $11.5 \pm 2.35 \mathrm{~nm}$ for OsMs with a $Ð$ of 0.03 (Supplementary Fig. 2a; Supplementary Table 1). Although micellar size usually increases after encapsulation of organic molecules ${ }^{39}$, incorporation of hydrophobic molecules can result in expulsion of water from the micelles, causing a contraction ${ }^{40}$. The hydrophobicity of 1 probably results in a stronger folding of the poly(ethylene oxide) chains around the complex through hydrophobic interactions, with concomitant expulsion of water from the core. A small second population of OsMs particles ( $<0.01 \%$ in number) was found at $D_{\mathrm{h}} \sim 220 \mathrm{~nm}$, exhibiting a strong intensity in DLS analysis, owing to the aggregation of some particles (Supplementary Fig. 2c).

Cryogenic TEM (cryo-TEM) analysis without staining was then performed on Quantifoil carbon-coated grids to observe the morphology of the hydrophobic core (containing osmium complexes) of the nanoparticles in solution. The high contrast provided by the heavy osmium centres allowed facile imaging of the osmiumpolypropylene glycol (PPG) core, but impaired the observation of the poly(ethylene glycol) corona owing to the polymer hydrated state; and disfavored by the small 


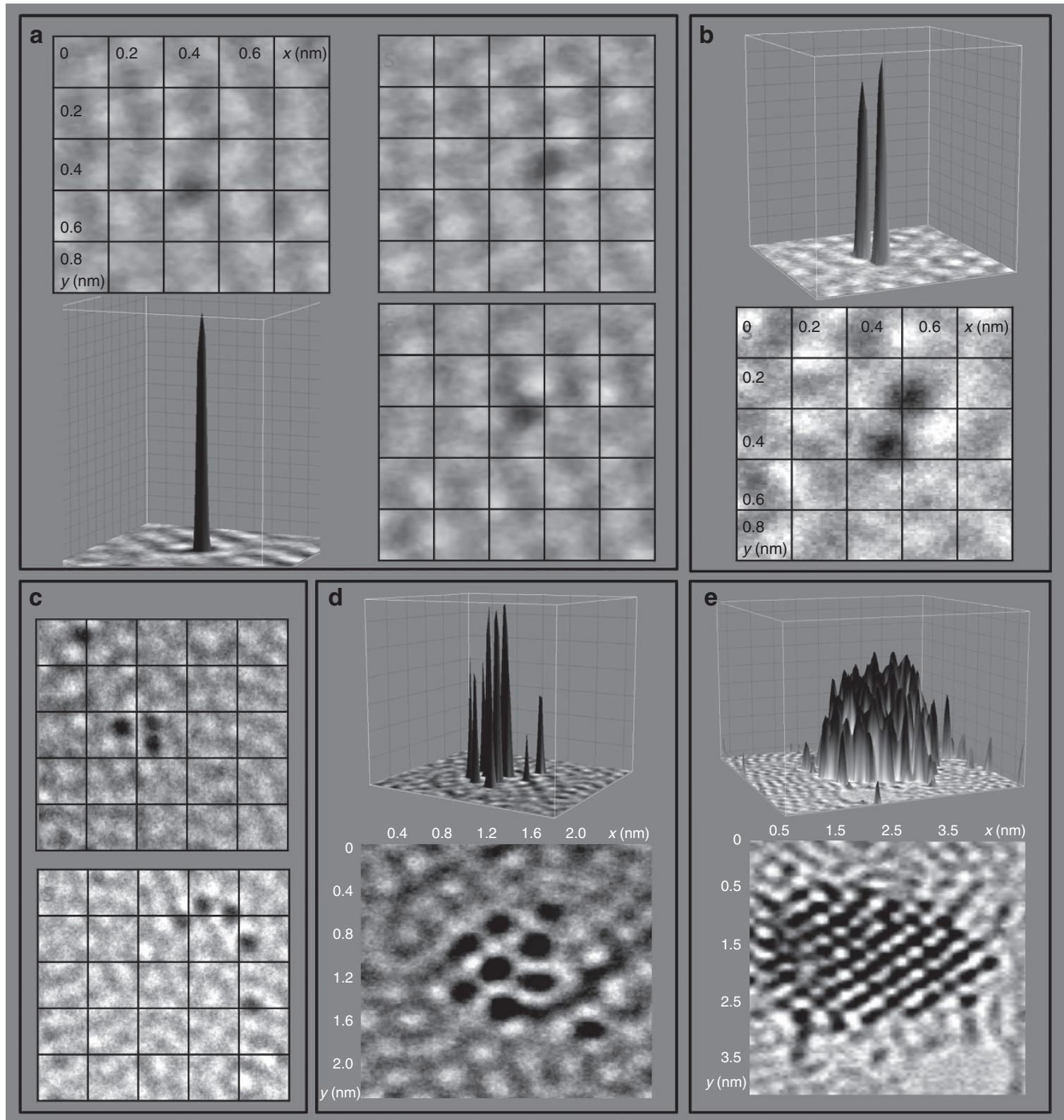

Figure 4 | Atom-by-atom formation of osmium molecules clusters and eventually crystals. (a) 3D projection showing high contrast for a single Os atom, together with three $2 \mathrm{D}$ projections showing movement of the Os atom at a rate of $0.0177 \mathrm{~nm} \mathrm{~s}^{-1}$ over a period of $160 \mathrm{~s}$. (b-c) Formation of dimer, trimer and tetramer molecules. (d) A cluster of eight Os atoms. (e) A ca. $25 \AA$ crystal. All the images show the same region.

diameter of the micelles (Supplementary Fig. 3a), even after attempts to further stain the samples with uranyl acetate. From these experiments, it was clear that spherical micellar morphologies are formed when polymer P123 encapsulates complex 1. The observed diameter of these OsMs nano-spheres is $7.85 \pm 1.97 \mathrm{~nm}$ (Supplementary Fig. 3b) with very low close-to-ideal dispersity, based on counting 157 particles counting $(1.06,1.00$ being for ideal mono-disperse systems; see Supplementary Table 1). These data are in accordance with the $D_{\mathrm{h}}$ determined by DLS within experimental error.

To gain further insight into their structures in aqueous solution, and to confirm cryo-TEM and DLS results, OsMs and P123Ms were analyzed by synchrotron SAXS (Supplementary Fig. 4). The experimental profiles were fitted to three model functions for spherical micelles: SphereForm, CoreShellSphere, and

PolyCoreShellRatio (PCR). The PCR model fitted excellently for both micelles with very low- $Ð$ parameters $(0.161$ for OsMs and 0.146 for P123Ms, 0 being an ideal mono-disperse system, Supplementary Table 1). These analyses demonstrated that OsMs self-assembly leads to core/shell micelles with a core diameter of $9.06 \pm 0.12 \mathrm{~nm}$, and a shell diameter of $6.50 \pm 0.15 \mathrm{~nm}$ (Supplementary Table 1). The core dimension of OsMs was larger than that of P123Ms $(6.74 \pm 0.06 \mathrm{~nm})$, while the corona dimension of OsMs was smaller than that of P123Ms shell $(12.22 \pm 0.17 \mathrm{~nm})$. The diameters of OsMs micelles by DLS and cryo-TEM are in accordance with the core diameter determined by SAXS within the experimental errors, while the diameters of P123 micelles from DLS and SAXS studies are similar.

From these data (scattering length density calculations, degrees of polymerization of Pluronic P123 and the molecular formulae of the polymer and of complex 1; see Instrumentation and Methods), aggregation numbers for OsMs and P123Ms micelles were determined as $20 \pm 2$ monomer chains per P123Ms micelle and $52 \pm 6$ monomer chains per OsMs micelles. Determinations of osmium by ICP-MS gave a polymer/complex 1 ratio of $1 / 1 \pm 0.091$ for OsMs showing that the 52 chains polymer chains self-assembled with $52 \pm 11$ complexes 1 (see Supplementary Table 1). Similar core/shell diameters (Supplementary Table 1) with excellent fits to the PCR model were obtained from experiments at three different concentrations $\left(1,5,10 \mathrm{mg} \mathrm{ml}^{-1}\right.$; Supplementary Fig. 4a,b) for both OsMs and P123Ms. Hence concentration does not influence the micellar structure, a parameter of importance for Pluronic-type self-assemblies.

\section{Instrumentation}

Ultraviolet-visible spectroscopy. Ultraviolet-visible absorption spectra were recorded on a temperature-controlled Varian CARY 300 Biospectrophotometer using $1-\mathrm{cm}$ path-length quartz cuvettes $(0.5 \mathrm{ml})$. 
Inductively coupled plasma mass spectrometry. Osmium $\left({ }^{189} \mathrm{Os}\right)$ content was determined using an ICP-MS Agilent technologies 7500 series instrument. The standard for osmium was purchased from Aldrich. Calibration curves were prepared using Os standard solutions in double deionised water with $3 \%$ nitric acid, ranging between 50 and $0.5 \mathrm{ppb}$ (9 points). Samples were freshly prepared in double deionised water with $3 \%$ nitric acid. Readings were made in no-gas mode with a detection limit of 1 ppt for ${ }^{189} \mathrm{Os}$.

Dynamic light scattering. The $D_{\mathrm{h}}$ of nanoparticles was determined by DLS. Typically, an aqueous nanoparticle solution was measured with a Malvern Zetasizer NanoS instrument equipped with a $4 \mathrm{~mW}$ He-Ne $633 \mathrm{~nm}$ laser module at $25^{\circ} \mathrm{C}$. Measurements were carried out at a detector angle of $173^{\circ}$ (back scattering). Data were analyzed by the Malvern DTS 6.20 software. $D_{\mathrm{h}}$ was calculated by fitting the apparent diffusion coefficient in the Stokes-Einstein equation $D_{\mathrm{h}}=k T /\left(3 \pi \eta D_{\mathrm{app}}\right)$, where $k$ is the Boltzmann constant, $T$ is the temperature and $\eta$ is the viscosity of the solvent. $D_{\mathrm{h}}$ coincides with the hydrodynamic diameter when the sample is made of monodispersed spherical particles ( $D_{\text {app }}$ equals the translational diffusion $D_{\mathrm{t}}$ ).

Transmission electron microscopy. TEM was performed using a JEOL 2000FX operating at $200 \mathrm{kV}$. TEM samples were prepared by using holey and lacey carbon grids. One drop of the sample solution $(5 \mu \mathrm{l})$ was applied to the grid and after $2 \mathrm{~min}$ the solution was blotted away before drying. Images were recorded on a Gatan Orius camera and were analyzed using ImageJ software. At least 100 particles from different parts of the grid were counted for each sample to obtain the average diameter.

Cryogenic-transmission electron microscopy. A JEOL 2010F TEM was operated at $200 \mathrm{keV}$ and images were recorded on a Gatan UltraScan 4000 camera for cryo-TEM and glow discharge. The samples were prepared at ambient temperature by placing a droplet on a TEM grid. The extra liquid was then blotted with a filter paper and the grid was inserted in liquid ethane at its freezing point. The frozen samples were subsequently kept under liquid nitrogen.

High-resolution electron microscopy. A JEOL JEM-ARM200F HR-TEM was operated at $80 \mathrm{keV}, 1.9 \mathrm{pA} \mathrm{cm}^{-2}$, with spherical aberration $\left(C_{\mathrm{s}}\right)$ tuned to approximately $+1 \mu \mathrm{m}$ and images were recorded on a Gatan SC1000 Orius CCD camera.

Small-angle X-ray scattering. Measurements were carried out on the SAXS beamline at the Australian Synchrotron facility at a photon energy of $11 \mathrm{keV}$. The samples in solution were in $1.5 \mathrm{~mm}$ diameter quartz capillaries. The measurements were collected at a sample to detector distance of $3.252 \mathrm{~m}$ to give a $q$ range of $0.004-0.2 \AA^{-1}$, where $q$ is the scattering vector and is related to the scattering angle $(2 \theta)$ and the photon wavelength $(\lambda)$ by the following equation (1)

$$
q=\frac{4 \pi \sin (\theta)}{\lambda}
$$

The scattering from a blank $\left(\mathrm{H}_{2} \mathrm{O}\right)$ was measured in the same location as sample collection and was subtracted for each measurement. All patterns were normalized to fixed transmitted flux using a quantitative beamstop detector. The scattering from a blank $\left(\mathrm{THF} / \mathrm{H}_{2} \mathrm{O}\right)$ was measured in the same location as sample collection and was subtracted for each measurement. The two-dimensional SAXS images were converted in one-dimensional SAXS profiles $(I(q)$ versus $q$ ) by circular averaging, where $I(q)$ is the scattering intensity. Functions were used from the NCNR package, using Igor software. Scattering length densities were calculated using the 'Scattering Length Density Calculator' provided by NIST Centre for Neutron Research.

\section{References}

1. Amoretti, M. et al. Production and detection of cold antihydrogen atoms. Nature 419, 456-459 (2002).

2. Arvizo, R. R. et al. Intrinsic therapeutic applications of noble metal nanoparticles: past, present and future. Chem. Soc. Rev. 41, 2943-2970 (2012).

3. Forster, S., Meinel, K., Hammer, R., Trautmann, M. \& Widdra, W. Quasicrystalline structure formation in a classical crystalline thin-film system. Nature 502, 215-218 (2013).

4. Ibáñez, M. \& Cabot, A. All change for nanocrystals. Science 340, 935-936 (2013).

5. Hunt, L. B. The true story of purple of Cassius. Gold Bull. 9, 134-139 (1976).

6. Faraday, M. Experimental relations of gold (and other metals) to light. Philos. Trans. R. Soc. London 147, 145-181 (1857).

7. Rao, C. N. R., Thomas, P. J. \& Kulkarni, G. U. Nanocrystals: Synthesis, Properties and Applications Vol. 95 (Springer-Verlag, 2007).

8. Qi, X. et al. In Situ modification of three-dimensional polyphenylene dendrimer-templated $\mathrm{CuO}$ rice-shaped architectures with electron beam irradiation. J. Phys. Chem. C 114, 13465-13470 (2010).

9. Zoberbier, T. et al. Interactions and reactions of transition metal clusters with the interior of single-walled carbon nanotubes imaged at the atomic scale. J. Am. Chem. Soc. 134, 3073-3079 (2012).
10. Bragg, W. L. Diffraction of X-rays by crystals. Nobel Lectures, Physics 1901-1921 (Elsevier Publishing Company, 1967).

11. Oh, M. H. et al. Galvanic replacement reactions in metal oxide nanocrystals. Science 340, 964-968 (2013).

12. Yamada, Y. et al. Nanocrystal bilayer for tandem catalysis. Nat. Chem. 3, 372-376 (2011).

13. Yang, J., Sargent, E., Kelley, S. \& Ying, J. Y. A general phase-transfer protocol for metal ions and its application in nanocrystal synthesis. Nat. Mater. 8, 683-689 (2009).

14. Langille, M. R., Personick, M. L. \& Mirkin, C. A. Plasmon-mediated syntheses of metallic nanostructures. Angew. Chem. Int. Ed. 52, 13910-13940 (2013).

15. Lara, P., Philippot, K. \& Chaudret, B. Organometallic ruthenium nanoparticles: a comparative study of the influence of the stabilizer on their characteristics and reactivity. Chem. Cat. Chem. 5, 28-45 (2013).

16. Xia, Y., Xiong, Y., Lim, B. \& Skrabalak, S. E. Shape-controlled synthesis of metal nanocrystals: simple chemistry meets complex physics? Angew. Chem. Int. Ed. 48, 60-103 (2009).

17. Lee, J., Zhou, W., Pennycook, S. J., Idrobo, J.-C. \& Pantelides, S. T. Direct visualization of reversible dynamics in a $\mathrm{Si}_{6}$ cluster embedded in a graphene pore. Nat. Commun. 4, 1650 (2013).

18. Muhich, C. L., Westcott, J. Y., Morris, T. C., Weimer, A. W. \& Musgrave, C. B. The effect of $\mathrm{N}$ and $\mathrm{B}$ doping on graphene and the adsorption and migration behaviour of Pt atoms. J. Phys. Chem. C 117, 10523-10535 (2013).

19. Zhang, T., Zhu, L., Yuan, S. \& Wang, J. Structural and magnetic properties of $3 \mathrm{~d}$ transition-metal-atom adsorption on perfect and defective graphene: a density functional theory study. Chem. Phys. Chem. 14, 3483-3488 (2013).

20. Lazar, P. et al. Quantification of the interaction forces between metals and graphene by quantum chemical calculations and dynamic force measurements under ambient conditions. ACS Nano 7, 1646-1651 (2013).

21. CRC. Handbook of Chemistry and Physics (CRC Press, 2011).

22. Chamberlain, T. W. et al. Formation of uncapped nanometre-sized metal particles by decomposition of metal carbonyls in carbon nanotubes. Chem. Sci. 3, 1919-1924 (2012).

23. Herberhold, M., Yan, H. \& Milius, W. The 16-electron dithiolene complexes (p-cymene) $\mathrm{M}\left[\mathrm{S}_{2} \mathrm{C}_{2}\left(\mathrm{~B}_{10} \mathrm{H}_{10}\right)\right](\mathrm{M}=\mathrm{Ru}, \mathrm{Os})$ containing both $\eta^{6}$-(p-cymene) and $\eta^{2}$-(ortho-carborane-dithiolate): adduct formation with Lewis bases, and $\mathrm{X}$-ray crystal structures of (p-cymene) $\mathrm{Ru}\left[\mathrm{S}_{2} \mathrm{C}_{2}\left(\mathrm{~B}_{10} \mathrm{H}_{10}\right)\right](\mathrm{L})\left(\mathrm{L}=\mathrm{PPh}_{3}\right)$ and $\left\{(\mathrm{p} \text {-cymene }) \mathrm{Ru}\left[\mathrm{S}_{2} \mathrm{C}_{2}\left(\mathrm{~B}_{10} \mathrm{H}_{10}\right)\right]\right\}_{2}(\mu-\mathrm{LL})\left(\mathrm{LL}=\mathrm{Ph}_{2} \mathrm{PCH}_{2} \mathrm{CH}_{2} \mathrm{PPh}_{2}\right.$ and $\left.\mathrm{N}_{2} \mathrm{H}_{4}\right)$ J. Organomet. Chem. 598, 142-149 (2000).

24. Scholz, M. \& Hey-Hawkins, E. Carbaboranes as pharmacophores: properties, synthesis, and application strategies. Chem. Rev. 111, 7035-7062 (2011).

25. Pitto-Barry, A. \& Barry, N. P. E. Pluronic ${ }^{\circledR}$ block-copolymers in medicine: from chemical and biological versatility to rationalisation and clinical advances. Polym. Chem. 5, 3291-3297 (2014).

26. Kiss, É., Keszthelyi, T., Kormány, G. \& Hakkel, O. Adsorbed and spread layers of poly(ethylene oxide) - poly(propylene oxide) - poly(ethylene oxide) block copolymers at the air - water interface studied by sum-frequency vibrational spectroscopy and tensiometry. Macromolecules 39, 9375-9384 (2006).

27. Suenaga, K. \& Koshino, M. Atom-by-atom spectroscopy at graphene edge. Nature 468, 1088-1090 (2010).

28. Wang, H. et al. Interaction between single gold atom and the graphene edge: A study via aberration-corrected transmission electron microscopy. Nanoscale 4, 2920-2925 (2012).

29. Online Dictionary of CRYSTALLOGRAPHY. http://reference.iucr.org/ dictionary/Crystal, Crystal Definition (2012).

30. Swanson, H. E., Fuyat, R. K. \& Ugrinic, G. M. Standard X-Ray Diffraction Powder Patterns in National Bureau of Standards Circular Vol. IV (1955).

31. Arblaster, J. W. Densities of Osmium and Iridium. Platinum Met. Rev. 33, 14-16 (1989)

32. Banhart, F., Kotakoski, J. \& Krasheninnikov, A. V. Structural Defects in Graphene. ACS Nano 5, 26-41 (2010).

33. Poh, H. L., Šimek, P., Sofer, Z. \& Pumera, M. Sulfur-doped graphene via thermal exfoliation of graphite oxide in $\mathrm{H}_{2} \mathrm{~S}, \mathrm{SO}_{2}$, or $\mathrm{CS}_{2}$ gas. ACS Nano 7 , 5262-5272 (2013).

34. Zhao, L. et al. Local atomic and electronic structure of boron chemical doping in monolayer graphene. Nano Lett. 13, 4659-4665 (2013).

35. Garcia, A. G., Baltazar, S. E., Castro, A. H. R., Robles, J. F. P. \& Rubio, A. Influence of $\mathrm{S}$ and $\mathrm{P}$ doping in a graphene sheet. J. Comput. Theor. Nanos $\mathbf{5}$, 2221-2229 (2008).

36. Cretu, O. et al. Migration and localization of metal atoms on strained graphene. Phys. Rev. Lett. 105, 196102 (2010).

37. Gan, Y., Sun, L. \& Banhart, F. One- and two-dimensional diffusion of metal atoms in graphene. Small 4, 587-591 (2008).

38. Rodriguez-Manzo, J. A. \& Banhart, F. Creation of individual vacancies in carbon nanotubes by using an electron beam of $1 \AA$ diameter. Nano Lett. 9 , 2285-2289 (2009). 
39. Jansson, J., Schillén, K., Olofsson, G., Cardoso da Silva, R. \& Loh, W. The interaction between PEO-PPO-PEO triblock copolymers and ionic surfactants in aqueous solution studied using light scattering and calorimetry. J. Phys. Chem. B 108, 82-92 (2003).

40. Parmar, A., Aswal, V. K. \& Bahadur, P. Interaction between the ionic liquids 1-alkyl-3-methylimidazolium tetrafluoroborate and Pluronic ${ }^{\mathbb{R}} \mathrm{P} 103$ in aqueous solution: a DLS, SANS and NMR study. Spectrochim. Acta A Mol. Biomol. Spectrosc. 97, 137-143 (2012).

\section{Acknowledgements}

We thank the Leverhulme Trust (Early Career Fellowship No. ECF-2013-414 to NPEB), the University of Warwick (Grant No. RDF 2013-14 to NPEB), the Swiss National Science Foundation (Grant No. PA00P2_145308 to NPEB and PBNEP2_142949 to APB), the ERC (Grant No. 247450 to PJS), EPSRC (EP/G004897/1 to RKOR, and EP/F034210/1 to PJS) and Science City (AWM/ERDF) for support. We thank the Wellcome Trust (Grant No. 055663/Z/98/Z) for funding the Electron Microscopy Facility, School of Life Sciences, University of Warwick. We also thank COST Action CM1105 for stimulating discussions, Thomas Wilks for supplying the micelle image for Figure 1, and the Australian Synchrotron and the University of Monash for allocation of time on the SAXS/ WAXS beamline and funding. The 2000FX Gatan Orius digital TEM camera used in this research was funded by Science City: Creating and Characterizing Next Generation Advanced Materials, with support from Advantage West Midlands and part funded by the European Regional Development Fund. We also thank Giovanni Costantini, Jeremy Sloan, Alessandro Troisi, Richard Walton and Neil Wilson for having kindly reviewed the manuscript prior to submission.

\section{Author contributions}

N.P.E.B. and P.J.S. conceived and obtained funding for the project, designed the experiments, oversaw the research and wrote the paper. R.B. and A.P.B. designed and performed the experiments, oversaw the research and wrote the paper. R.K.O.R. suggested key experiments, and discussed the results. A.P.D., R.J.P., J.J.S.B., A.M.S., N.K., I.H.P. and C.J.S. performed the experiments. All authors discussed the results and commented on the manuscript.

\section{Additional information}

Supplementary Information accompanies this paper at http://www.nature.com/ naturecommunications

Competing financial interests: The authors declare no competing financial interests.

Reprints and permission information is available online at http://npg.nature.com/ reprintsandpermissions/

How to cite this article: Barry, N. P. E. et al. Fabrication of crystals from single metal atoms. Nat. Commun. 5:3851 doi: 10.1038/ncomms4851 (2014).

(c) (i) This work is licensed under a Creative Commons Attribution 3.0 Unported License. The images or other third party material in this article are included in the article's Creative Commons license, unless indicated otherwise in the credit line; if the material is not included under the Creative Commons license, users will need to obtain permission from the license holder to reproduce the material. To view a copy of this license, visit http://creativecommons.org/licenses/by/3.0/ 